\title{
Macrocausality, Unitary and Physical-Region Structure of the Multiparticle $S$ Matrix
}

\author{
D. Iagolnitzer
}

Service de Physique Théorique, Cen-Saclay, F-91190 Gif-sur Yvette, France

\begin{abstract}
The physical-region analytic structure of the $S$-matrix is established on the basis of refined macrocausality, unitary and a weak supplementary analyticity assumption for a $3 \rightarrow 3$ process below the four-particle threshold, and away from points where two initial, or two final, four-momenta are colinear: the connected $S$ matrix is decomposed as a sum of contributions that generalize Feynman graphs and are associated with the various $+\alpha$-Landau surfaces encountered (= surfaces of graphs with one internal line and triangle graphs). The property of "separation of singularities" in unitarity equations, which was used as a crucial ad hoc assumption in traditional derivations of related results in $S$-matrix theory, is obtained as a byproduct in the region considered.
\end{abstract}

\section{Introduction}

This work is a continuation of recent works, carried out in the mathematical framework of essential support theory, or of hyperfunction theory, and aiming at the derivation in $S$-matrix theory, from basic principles, of the physical-region analytic structure of multiparticle collision amplitudes between sets of massive particles with short-range interactions.

Such a derivation was first attempted in the sixties [1] on the basis of unitarity and "maximal analyticity" [2], interpreted, when applied to the physical region, as providing analyticity of scattering functions (= connected momentum-space collision amplitudes, after factorization of their energy-momentum conservation $\delta$-functions) outside Landau surfaces of connected graphs, and the plus $i \varepsilon$ rule at $+\alpha$-Landau points. The aim was then to establish the detailed structure at $+\alpha$-Landau points, in the form of à la Cutkosky discontinuity formulae (with $S$-matrices associated to each vertex), as also analyticity outside the $+\alpha$-parts of the Landau surfaces. To that purpose, the general idea is to analyze the possible singularities of the various "bubble diagram functions" (=integrals, over internal on-mass-shell four-momenta, of products of connected kernels of $S$ or $S^{-1}=S^{\dagger}$ ) that occur in unitarity equations, and to use the fact that their singularities must cancel among themselves. 
While some basic ideas and problems were lucidly analyzed in [1], a number of other basic difficulties were, however, overlooked, and the proofs of [1] rely, as they stand, on assertions that were unproved and have turned out to be crucially non correct: see Appendix 2.1. For our purposes, we shall here consider the closely related program which starts from macrocausality $[3,4]$ and unitarity. Macrocausality provides (see [4]) a general essential support ${ }^{1}$ property of the connected $S$-matrix, which yields (and is essentially equivalent to) analyticity outside $+\alpha-$ Landau surfaces of connected graphs and the plus $i \varepsilon$ rule at $+\alpha$-Landau points. It gives also information at points where the plus is rule cannot be expected ${ }^{2}$. The aim is then, as above, to use unitarity equations in order to derive discontinuity formulae and related results.

The derivations given until recently in that approach, at the end of the sixties [10], or in a more complete and satisfactory way in the seventies [11-13], make a crucial use, however, of an ad hoc assumption of "separation of singularities" in unitarity equations, according to which there is independent cancellation of singularities corresponding to diagrams $\mathscr{D}_{B}$ (see Appendix 1) with related topological structure (see illustration in Appendix 2.2). The property of separation of singularities is believed to hold, but it has no à priori basis and should thus itself be derived rather than be used as an assumption.

A first basic difficulty that appears is then the " $u=0$ problem" (see Appendix 1) which prevents one à priori to get any information on terms such as $(+)-(-)$. It has been solved in the framework of essential support theory in [9], where a $u=0$ theorem $^{3}$ is proved on the basis of a refined version of the macrocausality condition (= regularity property $R$ ). A related $u=0$ conjecture was also proposed in [8] on the basis of general results on phase-space integrals. See also the recent results of [14] on products of holonomic functions with regular singularities.

Even though the $u=0$ problem is solved, other apparently crucial problems remain. The information obtained on each bubble diagram function is its essential support, i.e. the location of its singularities. (We mean here both the singularities in momentum-space and the singular directions at each real singular point.) Being given bubble diagram functions whose singularities correspond to diagrams with non related topological structures, one might hope that the location of their singularities would be such that separation of singularities would be automatically guaranteed, when needed, by the general arguments of essential support, or

\footnotetext{
1 The essential support of a distribution $f$ is, at each real point $P$, a cone composed of "singular directions" along which a generalized Fourier transform of $f$ at $P$ does not fall of exponentially in a certain sense. It specifies by duality the real points where $f$ is analytic, or is the boundary value of an analytic function, and characterizes more generally possible decompositions of $f$ into sums of boundary values of analytic functions. For details and further results, see $[5,4]$. The essential support of $f$ coincides [6] with its singular spectrum, introduced independently, by different methods, in hyperfunction theory [7]. By definition, $f$ is micro-analytic at $P$ in a direction $\hat{u}$, if $\hat{u} \notin S S_{P}(f) \equiv E S_{P}(f)$ 2 I.e. points that lie at the intersection of several $+\alpha$-Landau surfaces with different plus $i \varepsilon$ directions, and the $\mathscr{M}_{0}$ points $p=\left\{p_{k}\right\}$ such that some initial, or some final, $p_{k}$ are colinear. (For a discussion of the essential support at $\mathscr{M}_{0}$ points, see $[8,9]$.) The above points, although exceptional, are always encountered in integration domains in the study of bubble diagram functions

3 The $u=0$ theorem ensures that the essential support at $u=0$ points is determined as previously, i.e. (see Appendix 1) by the configurations of external trajectories of diagrams $\mathscr{D}_{B}$, except, however, that it may be enlarged in general by some limiting procedures
} 
hyperfunction, theory (=generalizations of the edge-of-the-wedge theorem). But this is not the case, and previous derivations are à priori completely disrupted (see Appendix 2), as explained in detail in [15], if separation of singularities is not assumed.

A solution in several steps has been obtained in [15] in the case of the derivation of the pole-factorization theorem (= structure of the $S$ matrix in the neighborhood of points of $+\alpha$-Landau surfaces of graphs with one internal line), for a $3 \rightarrow 3$ process below the four-particle threshold. The derivation of [15] makes use, besides macrocausality and unitarity, of an ad hoc $u=0$ assumption, which can be removed by the results of [9], and of a "no sprout assumption", which goes slightly beyond the analyticity properties derived for scattering functions from macrocausality. It is linked with, and can probably be derived from, refined macrocausality: see [9]. It is also a weak aspect of possible assumptions of "maximal analyticity", or of holonomy.

The present work makes use of the tools and results of [15], but introduces a somewhat different method, based on an adequate grouping, at the various stages of the derivation, of singular contributions to the various bubble diagram functions that can be expected (by the general idea of separation of singularities), and will here be proved, to cancel among themselves. This method leads to a simplified version of the work of [15] and is used here to derive the complete physical-region structure of the $S$-matrix for a $3 \rightarrow 3$ process below the fourparticle threshold (and away from $\mathscr{M}_{0}$ points - see definition in Footnote 2). A no sprout assumption which is an extension of that of [15], and to which comments similar to above apply, will be needed. As explained in Sect. 2.1, macrocausality entails that the connected $S$-matrix decomposes in the region considered as a sum of contributions associated with the various $+\alpha$-Landau surfaces $L^{+}(G)$ encountered ( $=$ surfaces of graphs with one internal line and triangle graphs), each of which being analytic outside the corresponding surface $L^{+}(G)$ and being the plus $i \varepsilon$ boundary value of an analytic function along $L^{+}(G)$. The no sprout assumption, stated in Sect. 2.1, excludes certain à priori possible pathologies of these contributions. It is then established in Sect. 3 that they are necessarily generalizations, defined in Sect. 2.2, of the Feynman integrals associated with the graphs $G$, whose discontinuities are given by the à la Cutkosky formulae already mentioned. (For their links with field theory, see Sect. 2.2.)

Separation of singularities is established in the course of the proof when needed. It follows in more general cases, in the region considered, from the results of Sect. 3: see Appendix B of [13].

The structure theorem of [11] on bubble diagram functions, which is used several times in Sects. 2 and 3, is briefly recalled in Appendix 1.

Previous derivations of the pole-factorization theorem in $S$-matrix theory are outlined, and compared to the present one, in Appendix 2.

Finally, possible extensions of the results are briefly mentioned and discussed in Appendix 3.

\section{Notes}

1. It is known (see $[13,16]$ ) that results such as those of the present work can alternatively be derived from, and are in fact equivalent to properties of macro- 
causality and macrocausal factorization. The latter is a factorization property of transition amplitudes for causal configurations of displaced particles, whereas macrocausality gives information for non causal configurations. However, it is not likely to be independent, and it is desirable to derive it if possible, from the more basic principles of macrocausality and unitarity.

2 . Results closely related to those of Sect. 3 , and applying also to $3 \rightarrow 3$ processes below the four-particle threshold, have been recently obtained in axiomatic field theory, in two different approaches $[17,18]$. Information on the essential support of the multiparticle $S$-matrix at physical-region points was derived previously [19] from microcausality and the spectral condition. It is, however, much weaker than macrocausality. This is compensated in $[17,18]$ by the exploitation of the off-shell structure of field theory and of the axiom of asymptotic completeness, together with regularity assumptions. The latter are needed to avoid à priori possible à la Martin pathologies ${ }^{4}$.

\section{Preliminary Definitions and Results}

\subsection{The No Sprout Assumption}

We consider a theory with one type of particle, a spinless boson of mass $\mu>0$, and a process with three initial final particles labelled by the respective indices $1,2,3$, and 4, 5, 6. The initial and final energy-momenta 4-vector variables are denoted by $p_{k}=\left(\left(p_{k}\right)_{0}, \mathbf{p}_{k}\right), k=1, \ldots, 6$. The physical region $\mathscr{M}$ of the process is the set of points $p=\left(p_{1}, \ldots, p_{6}\right)$ that satisfy the mass-shell constraints $\left(p_{k}^{2}=\left(p_{k}\right)_{0}^{2}\right.$ $\left.-\mathbf{p}_{k}^{2}=\mu^{2},\left(p_{k}\right)_{0}>0, k=1, \ldots, 6\right)$ and energy-momentum conservation $\left(p_{1}+p_{2}+p_{3}\right.$ $\left.=p_{4}+p_{5}+p_{6}\right)$. The scattering function $f$ of the process is the well defined distribution on $\mathscr{M}$ (away from points $p$ such that all $p_{k}$ are colinear) obtained from the connected $S$-matrix kernel after factorization of the $\delta^{4}$ function of energymomentum conservation it contains.

The part $\mathscr{R}$ of the physical region we shall be mainly interested in is defined by the equations $(3 \mu)^{2}<s=\left(p_{1}+p_{2}+p_{3}\right)^{2}<(4 \mu)^{2}$ and $p \notin \mathscr{M}_{0} . \mathscr{M}_{0}$ is the set of $p$ such that two initial, or two final, $p_{k}$ are colinear (and hence equal in the present equal-mass case). In the region $\mathscr{R}$, eighteen $+\alpha$-Landau surfaces $L^{+}\left(G_{\beta}\right)$ of connected graphs, which are unrelated, ${ }^{5}$ are encountered. They correspond to nine graphs with one internal line, such as $G_{1}=4$ graphs, such as $G_{2}=$ codimension one submanifolds in $\mathscr{R}$. For any given $G_{\beta}$ and any point $P=\left(P_{1}, \ldots, P_{6}\right)$ of $\mathscr{R}$ that lies on $L^{+}\left(G_{\beta}\right)$, there exists correspondingly only one classical multiple scattering diagram $\mathscr{D}_{+}\left(P ; G_{\beta}\right)$ in space-time whose topological structure is $G_{\beta}$ and whose external trajectories have the four-momenta $P_{k}$, modulo global spacetime translations and dilations. This is still true for each $\beta$ in the region $\mathscr{R}_{\beta}$ obtained

4 The exclusion of these pathologies is related to certain physical conditions (no accumulation of an infinite number of unstable particles with arbitrarily small widths) which are also part of the conditions upon which macrocausality is based

5 I.e. there is no point $P$ in $\mathscr{R}$ that belongs to several surfaces $L^{+}\left(G_{\beta}\right)$ (or their closures) of graphs $G_{\beta}$ that are all contractions of one of them 
by removing only, from the region $(3 \mu)^{2}<s<(4 \mu)^{2}$, the points $p$ that belong to $L^{+}\left(G_{\beta}\right)$ and are such that $p_{1}=p_{2}$ or $p_{5}=p_{6}$ in the case of the graph $G_{1}, p_{1}=p_{2}$ or $p_{4}=p_{5}$ in the case of $G_{2}$.

The direction in the cotangent space $T_{P}^{*} \cdot \mathscr{U}$ at $P$ to $\mathscr{M}$ determined by the relative configuration of external trajectories of $\mathscr{L}_{+}\left(P ; G_{\beta}\right)$ will be denoted $\hat{u}_{+}\left(P ; G_{\beta}\right)$. The opposite direction is denoted $\hat{u}_{-}\left(P ; G_{\beta}\right)$.

The following proposition is a direct consequence of macrocausality and of the decomposition theorems of essential support theory.

Proposition 1. "There exists in the region $(3 \mu)^{2}<s<(4 \mu)^{2}$ decompositions of $f$ of the form:

$$
f=\sum_{\beta} f_{\beta}+a
$$

where $a$ is analytic outside $\mathscr{M}_{0}$ points and where, for each $\beta$,

$$
\begin{array}{lll}
E S_{P}\left(f_{\beta}\right) \subset\left\{\hat{u}_{+}\left(P ; G_{\beta}\right)\right\} & \text { if } & P \in L^{+}\left(G_{\beta}\right) \cap \mathscr{R}_{\beta}, \\
E S_{P}\left(f_{\beta}\right)=\{0\} & \text { if } & P \notin L^{+}\left(G_{\beta}\right),
\end{array}
$$

Correspondingly, $f_{\beta}$ is analytic outside $L^{+}\left(G_{\beta}\right)$ and, is at each point $P$ of $L^{+}\left(G_{\beta}\right)$ in $\mathscr{R}_{\beta}$, the boundary value, from the "plus i $\varepsilon$ " directions dual to $\hat{u}_{+}\left(P ; G_{\beta}\right)$, of a function $\underline{f}_{\beta}$ analytic in a domain of the complexified manifold $\underline{M}$ of $\mathscr{M} . "$

Proposition 1 can be completed in a natural way by specifying also the essential support of $a$ at $\mathscr{M}_{0}$ points and of $f_{\beta}$ at points of $L^{+}\left(G_{\beta}\right)$ outside $\mathscr{R}_{\beta}$. These informations will, however, not be needed for our later purposes and have thus been omitted.

The decomposition (1) of $f$ is not unique, even in $\mathscr{R}$. Besides the obvious fact that analytic functions $a_{\beta}$ can be added to each $f_{\beta}$, there are also à priori more serious ambiguities, which will be excluded only (see Lemma 1 below) by the no sprout assumption. This problem turns out, for reasons explained below, to play an important role and we thus discuss it in more detail. The ambiguities arise from the occurrence of points $P$ that lie on two surfaces $L^{+}\left(G_{\beta_{1}}\right)$ and $L^{+}\left(G_{\beta_{2}}\right)$ that are tangent at $P$ and are such that $\hat{u}_{+}\left(P ; G_{\beta_{1}}\right) \equiv \hat{u}_{+}\left(P ; G_{\beta_{2}}\right)$. It is then possible to add to $f_{\beta_{1}}$, and substract from $f_{\beta_{2}}$, any $f^{\prime}$ which is for instance analytic in $\mathscr{R}$ minus $P$ and is such that $E S_{P}\left(f^{\prime}\right)=\left\{\hat{u}_{+}\left(P ; G_{\beta_{1}}\right)\right\}, i=1$, 2. (Such terms $f^{\prime}$, singular only at $P$, do exist.) Points $P$ as above are for instance those of the subset $\Omega\left(G_{2}\right)$ of $L^{+}\left(G_{2}\right)$ such that the four-momenta of the two internal trajectories of $\mathscr{D}_{+}\left(P ; G_{2}\right)$, incoming at the vertex where 4, 5 are outgoing, are equal to $P_{4}$ and $P_{5}{ }^{6}$ As easily seen, all points of $\Omega\left(G_{2}\right)$ belong also to $L^{+}\left(G_{1}\right)$ (and as a matter of fact to the subset $\Omega_{+}$of $L^{+}\left(G_{1}\right)$ defined in [15]) and $\hat{u}_{+}\left(P ; G_{1}\right)=\hat{u}_{+}\left(P, G_{2}\right)$ there: the topological structure of $\mathscr{D}_{+}\left(P ; G_{1}\right)$ and $\mathscr{D}_{+}\left(P ; G_{2}\right)$ is different [there is a supplementary vertex in $\left.\mathscr{D}_{+}\left(P ; G_{2}\right)\right]$, but their configurations of external trajectories coincide.

$6 \Omega\left(G_{2}\right)$ is not empty. In fact, if one considers any point $P^{\prime}=\left(P_{1}^{\prime}, \ldots, P_{6}^{\prime}\right)$ of $L^{+}\left(G_{2}\right)$ in $\mathscr{R}_{2}$, the internal on-mass-shell four-momenta in $\mathscr{D}_{+}\left(P^{\prime} ; G_{2}\right)$ are well determined. Let $K_{1}, K_{2}$ be those of the lines that are incoming at the vertex where 4,5 are outgoing. Then the point $\left(P_{1}^{\prime}, P_{2}^{\prime}, P_{3}^{\prime}, P_{4}=K_{1}, P_{5}=K_{2}, P_{6}^{\prime}\right)$ belongs to $\Omega\left(G_{2}\right)$ 
Even though they lie in low-dimensional submanifolds, such points cannot be avoided. This is because, if one considers the bubble diagram function ${ }_{3}^{1}=(+)_{-}^{1} \Theta_{6}^{4}$ then given any $P$ on $L^{+}\left(G_{2}\right)$, such points always occur for the bubble $\equiv+\Phi^{+}$in the integration domain. For that reason, we shall need, in Sect. 3, the no sprout assumption in the form stated below, which, in contrast to that given in [15], covers these points. We first recall the definition of the no sprout property. (A weaker form of this property would also be sufficient: see [15].)

Let $h_{\beta}$ be a distribution satisfying in $\mathscr{R}_{\beta}$ (or possibly in a subdomain $\mathscr{R}_{\beta}^{\prime}$ of $\mathscr{R}_{\beta}$ containing $\left.L^{+}\left(G_{\beta}\right) \cap \mathscr{R}_{\beta}\right)$ the essential support properties (2), (3) or the equivalent analyticity properties stated below (2), (3) for $f_{\beta}$. Let $q=\left(q_{1}, \ldots, q_{14}\right)$ be a system of real analytic local coordinates of $\mathscr{M}$ at a point $P$ of $L^{+}\left(G_{\beta}\right)$ in $\mathscr{R}_{\beta}$, chosen such that $L^{+}\left(G_{\beta}\right)$ is locally defined, in the neighborhood of $P$, by $\ell(q)=q_{1}=0$ and such that $\hat{u}_{+}\left(P ; G_{\beta}\right)$ is the direction of the $u_{1}$-axis. The above mentioned essential support or analyticity properties of $h_{\beta}$ guarantee that, being given any open cone $\Gamma$ with its apex at the origin in $\operatorname{Im} q$-space (where $q$ is the complexified variable of $q$ ), whose closure is contained (apart from the origin) in the region $\operatorname{Im} \underline{\ell}=\operatorname{Im} \underline{q}_{1}>0$, there exists a complex neighborhood $\Omega$ of $q=0(\equiv P)$ such that $\underline{h}_{\beta}$ is analytic in $\Omega \cap\{\operatorname{Im} q \in \Gamma\}$. However, $\underset{\Omega}{\Omega}$ might a priori shrink to zero when $\Gamma$ expands to the whole half-space $\operatorname{Im} \underline{\ell}>0$.

Definition. " $h_{\beta}$ satisfies the no sprout property at $P$ if there exists a complex neighborhood $\underset{\Omega}{\Omega}$ of $P$ such that $\underline{h}_{\beta}$ is analytic in $\underset{\sim}{\Omega} \cap\{\operatorname{Im} \underline{\ell}>0\}$."

No Sprout Assumption. "There exists a decomposition of the form (1) of $f$ such that each $f_{\beta}$ satisfies the no sprout property at each point $P$ of $L^{+}\left(G_{\beta}\right)$ in $\mathscr{R}_{\beta}$."

This assumption is natural in the neighborhood of points $P$ that belong to two surfaces $L^{+}\left(G_{\beta_{1}}\right)$ and $L^{+}\left(G_{\beta_{2}}\right)$ such that $\hat{u}_{+}\left(P ; G_{\beta_{1}}\right)=\hat{u}_{+}\left(P ; G_{\beta_{2}}\right)$ because the two surfaces are unrelated at $P$ (see definition in Footnote 5).

Lemma 1. "The decomposition (1) of $f$ provided by the no sprout assumption is unique in $\mathscr{R}$, modulo addition to each $f_{\beta}$ of a function $a_{\beta}$ analytic in $\mathscr{R} . "$

Lemma 1 is a direct consequence of Lemma 2 below, which will also be useful in Sect. 3. Being given two decompositions $f=\sum f_{\beta}+a$ and $f=\sum f_{\beta}^{\prime}+a^{\prime}$, it is obtained by applying, for each $\beta$, Lemma 2 to $h_{\beta}=f_{\beta}-f_{\beta}^{\prime}$. The equality $f_{\beta}-f_{\beta}^{\prime}$ $=\sum_{\beta^{\prime} \neq \beta}\left(f_{\beta^{\prime}}^{\prime}-f_{\beta^{\prime}}\right)+a^{\prime}-a$ guarantees in fact the analyticity of $f_{\beta}-f_{\beta}^{\prime}$ almost everywhere along $L^{+}\left(G_{\beta}\right)$.

Lemma 2. Let $h_{\beta}$ satisfy the essential support properties (2), (3) in $\mathscr{R}_{\beta}$ (resp. $\left.\mathscr{R}_{\beta}^{\prime}\right)$, and the no sprout property at each point $P$ of $\mathscr{R}_{\beta}$ (resp. of $\left.\mathscr{R}_{\beta}^{\prime}\right)$. If $h_{\beta}$ is analytic at one point $P$ of $L^{+}\left(G_{\beta}\right)$, it is necessarily analytic in the whole region $\mathscr{R}_{\beta}\left(\right.$ resp. $\left.\mathscr{R}_{\beta}^{\prime}\right)$.

In other words, $h_{\beta}$ cannot be analytic at some points, and singular at other points, of $L^{+}\left(G_{\beta}\right)$ if it satisfies the no sprout property. Lemma 2 follows immediately from Theorem 1 of Sect. 5 of [15], itself a direct consequence of Bremmerman's continuity theorem. 


\subsection{Generalized Feynman Integrals}

Being given a graph $G_{\beta}$, let $D_{\beta}$ be the bubble diagram function obtained by replacing its vertices by plus bubbles. For instance:

$$
\begin{aligned}
D_{2} & ={ }_{3}^{1}={ }_{+\oplus}^{\oplus}{ }_{5}^{4} \\
& \equiv \int_{2,2}^{c}\left(p_{1}, p_{2} ; k_{1}, k_{2}\right) S_{2,2}^{c}\left(k_{2}, p_{3} ; k_{3}, p_{6}\right) S_{2,2}^{c}\left(k_{1}, k_{3} ; p_{4}, p_{5}\right) \\
& \cdot \prod_{t=1,2,3} \delta\left(k_{\ell}^{2}-\mu^{2}\right) \theta\left(\left(k_{\ell}\right)_{0}\right) d^{4} k_{\ell} .
\end{aligned}
$$

We shall denote by $d_{\beta}$ the distribution defined on $\mathscr{M}$ from $D_{\beta}$ by factorizing out its $\delta^{4}$-function $\delta^{4}\left(p_{1}+p_{2}+p_{3}-p_{4}-p_{5}-p_{6}\right)$, and by $d_{\beta}^{+}$the distribution, well defined modulo an analytic function in $\mathscr{R}_{\beta}$ (see below), whose discontinuity around $L^{+}\left(G_{\beta}\right)$ is $d_{\beta}$ and which is analytic in $\mathscr{R}_{\beta}$ outside $L^{+}\left(G_{\beta}\right) ; d_{\beta}^{+}$can be defined here as follows. First, one checks easily from the structure theorem and remark (ii) in Appendix 1 that $E S_{p}\left(d_{\beta}\right)$ is contained, at any point $P$ in $\mathscr{R}_{\beta}$, in the set $C\left(P ; G_{\beta}\right)$ determined by relative configurations of external trajectories of diagrams $\mathscr{D}\left(P ; G_{\beta}\right)^{7}$. Let $C_{+}\left(P ; G_{\beta}\right)$ and $\hat{C}\left(P ; G_{\beta}\right)$ be the sets determined by diagrams $\mathscr{D}_{+}\left(P ; G_{\beta}\right)$ and other diagrams $\mathscr{D}\left(P ; G_{\beta}\right)$ respectively. $C_{+}$is composed of the direction $\hat{u}_{+}\left(P ; G_{\beta}\right)$ if $P \in L^{+}\left(G_{\beta}\right)$ and is empty otherwise; $\hat{C}$ is composed of $\hat{u}_{-}\left(P, G_{\beta}\right)$ if $P \in L^{+}\left(G_{\beta}\right)$. If $G_{\beta}$ is a triangle graph, $\hat{C}$ is not empty everywhere outside $L^{+}\left(G_{\beta}\right)$. However $C_{+}$and $\hat{C}$ remain disjoint and Lemma 10 of Sect. $C$ of [4] yields decompositions of $d_{\beta}$ of the form:

$$
d_{\beta}=d_{\beta}^{+}+\hat{d}_{\beta},
$$

where $d_{\beta}^{+}$and $\hat{d}_{\beta}$ are well defined modulo analytic functions in $\mathscr{R}_{\beta}$, and where their essential supports are contained respectively, at each $P$ in $\mathscr{R}_{\beta}$, in the sets $C_{+}$and $\hat{C}$.

We shall put $D_{\beta}^{+}=d_{\beta}^{+} \times \delta^{4}\left(p_{1}+p_{2}+p_{3}-p_{4}-p_{5}-p_{6}\right)$, and write it diagrammatically in the same way as $D_{\beta}$, but with + signs above each internal line. These signs may remind the reader that the essential supports of $d_{\beta}$ and $d_{\beta}^{+}$correspond respectively, as explained above, to diagrams $\mathscr{D}$ and $\mathscr{D}_{+}$: the vertex to which any internal trajectory of $\mathscr{D}_{+}$, associated with any internal line of $D_{\beta}^{+}$is incoming is always later in time than the vertex at which it is outgoing, whereas there is no such time ordering condition in the diagrams $\mathscr{D}$. These signs also refer to the fact that $d_{\beta}^{+}$is a natural physical region generalization, modulo analytic backgrounds, of the Feynman integral associated with $G_{\beta}$ : in view of Cutkosky's discontinuity formula, the latter is indeed equal, modulo an analytic function, to the term analogous to $d_{\beta}^{+}$obtained when the bubbles are constants, times conservation $\delta$-functions, rather than actual $S$-matrix kernels.

The distribution $d_{\beta}^{+}$can be itself represented explicitly as a Feynman type integral in which the mass-shell $\delta$-functions $\delta\left(k_{\ell}^{2}-\mu^{2}\right)$ [see (4)] are replaced by Feynman propagators $\left(k_{\ell}^{2}-\mu^{2}+i \varepsilon\right)^{-1}$. More precisely, if $G_{\beta}$ is e.g. the graph $G_{1}$, one has:

$$
d_{\beta}^{+}=\frac{b\left(p_{1}, \ldots, p_{6}\right)}{k^{2}-\mu^{2}+i \varepsilon}
$$

7 Diagrams $\mathscr{D}$ are defined in the same way as diagrams $\mathscr{D}_{+}$except that the time-ordering conditions on the vertices are removed. They may include vertices at infinity (see $[8,9])$ 
where $k=p_{1}+p_{2}-p_{4}$ and $b$ is any function analytic in $\mathscr{R}_{\beta}$, whose residue at $k^{2}=\mu^{2}$ is the product $f_{2,2}\left(p_{1}, p_{2} ; p_{4}, k\right) f_{2,2}\left(k, p_{3} ; p_{5}, p_{6}\right)$. For triangle graphs, off-shell extensions of the scattering function $f_{2,2}$ and cut-off factors have to be introduced in the integrand. We shall not discuss here this procedure, which is not natural in the present framework and would require a more detailed analysis: see Theorem 2.1.4 of [20] for a micro-local study. We note on the other hand that generalizations of Feynman integrals, or $G$-convolutions, can be defined in field theory by replacing each vertex by an $n$-point function and have been extensively studied by J. Bros and coworkers. It can be checked (see $[17,18]$ ) that they do coincide in the physical region, modulo analytic backgrounds, with the $d_{\beta}^{+}$introduces here. For their general analyticity properties, see [17].

\section{The Physical-Region Three-Body $S$-Matrix}

Theorem. Refined macrocausality, unitarity and the no sprout assumption imply the following decomposition in $\mathscr{R}$ :

$$
f=\sum_{\beta} d_{\beta}^{+}+r
$$

or in a diagrammatical notation (see Sect. 2.2):

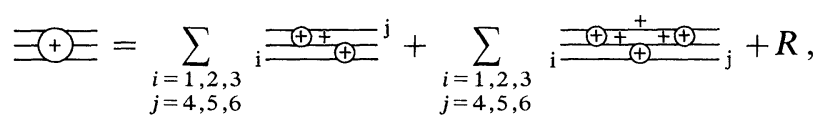

where $R=r \times \delta^{4}\left(p_{1}+p_{2}+p_{3}-p_{4}-p_{5}-p_{6}\right)$ and $r$ is analytic in $\mathscr{R}$.

The statement is clearly independent of the choice of the terms $d_{\beta}^{+}$, which are defined only modulo analytic functions in $\mathscr{R}$.

We consider a decomposition $f=\sum f_{\beta}+a$, provided by macrocausality, in which each $f_{\beta}$ satisfies the no sprout property (see no sprout assumption), and will show that for each $\beta$, one has necessarily $f_{\beta}=d_{\beta}^{+}$in $\mathscr{R}_{\beta}$, modulo an analytic function.

Unitarity will be used in the form of the following well-known equation below the four-particle threshold:

$$
\begin{aligned}
& -\exists+E+\exists(E+\exists+E E+\exists+E(-)
\end{aligned}
$$

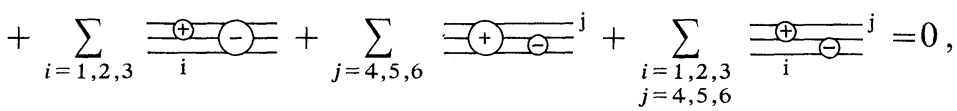

where the plus bubbles represent as previously connected $S$-matrix kernels and the minus bubbles, according to a usual convention, represent here connected kernels of $S^{-1}=S^{\dagger}$, multiplied by -1 .

\subsection{Graphs with One Internal Line}

We first consider a graph with one internal line, for instance $G_{1}=$ and then rewrite Eq. (10) in the form:

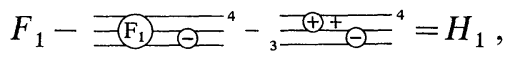


where $F_{\beta}=f_{\beta} \times \delta^{4}\left(p_{1}+p_{2}+p_{3}-p_{4}-p_{5}-p_{6}\right)$, ${\stackrel{\oplus+}{\Theta}{ }^{4}}^{-}$is defined as $D_{1}^{+}$in Sect. 2.2 modulo an analytic function in $\mathscr{R}_{1}$ (the change of the plus bubble on the right by a minus bubble does not affect the main facts), and $H_{1}$ is the sum of all

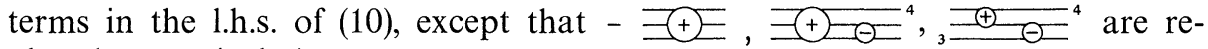
placed respectively by

and

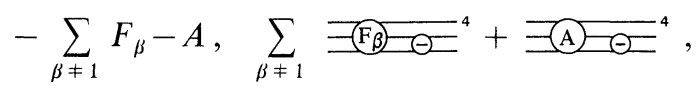

where $A=a \times \delta^{4}\left(p_{1}+p_{2}+p_{3}-p_{4}-p_{5}-p_{6}\right)$.

As follows from the structure theorem (see Appendix 1), the two terms singled out with $F_{1}$ in the 1.h.s. of (11) are those whose singularities correspond to diagrams with a related topological structure. They are here, like $f_{1}$, analytic outside $L^{+}\left(G_{1}\right)$ and have only $\hat{u}_{+}\left(P ; G_{1}\right)$ in their essential support at any point $P$ of $L^{+}\left(G_{1}\right)$ in $\mathscr{R}_{1}$ (after factorization of their $\delta^{4}$-functions).

By "multiplication" on the right of all terms of (11) by $\overline{\overline{1}}^{4}$ (in the sense of on-mass-shell convolutions, i.e. each term where $工+工$ is the non connected $S$-matrix kernel, and by two-particle unitarity below the three-particle threshold $(=\odot=-\Theta--\exists+=-$ - $(11)$ is transformed into:

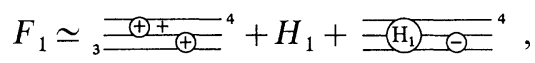

where the $\operatorname{sign} \simeq$ means that both sides are equal in $\mathscr{R}_{1}$ modulo an analytic function (after factorization of $\delta^{4}$-functions).

In fact, the sum of the first two terms in the 1.h.s. of (11) is clearly transformed into $F_{1}$ and the following simple lemma will be proved at the end of this subsection:

\section{Lemma 3}

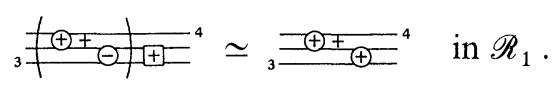

In view of (12), the desired result will then be achieved if one can prove that

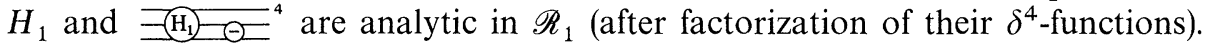
This is achieved in steps (i)-(iii).

(i) The structure theorem (see Appendix 1), the $u=0$ theorem of [9] (applied to $(+)-(-)$ ) and a detailed analysis of all terms included in $H_{1}$, which is essentially the same as in [15] up to minor changes, allows one to conclude that $\hat{u}_{+}\left(P ; G_{1}\right)$ $\notin E S_{P}\left(h_{1}\right)$ if $P$ lies outside an open subsect $\Omega_{+}$of $L_{+}\left(G_{1}\right)$ and outside the union $\mathscr{N}$ of certain other lower-dimensional submanifolds of $L^{+}\left(G_{1}\right) .(\mathscr{N}$ is here slightly different from the corresponding set in [15].) We have put $H_{1}=h_{1} \times \delta^{4}\left(p_{1}+p_{2}+p_{3}\right.$ $\left.-p_{4}-p_{5}-p_{6}\right)$.

In view of the equality (11) and of the fact that the terms in the 1.h.s. of (11) have at most $\hat{u}_{+}\left(P ; G_{1}\right)$ in their essential support at any $P$ of $L^{+}\left(G_{1}\right)$ in $\mathscr{R}_{1}, E S_{P}\left(h_{1}\right)$ is empty, i.e. $h_{1}$ is analytic, if $P \notin \Omega_{+} U \mathscr{N}$. The equality (11) entails similarly that $h_{1}$ is analytic outside $L^{+}\left(G_{1}\right)$.

(ii) The terms in the 1.h.s. of (11), after factorization of their $\delta^{4}$-functions, and hence $h_{1}$, satisfy the no sprout property along $L^{+}\left(G_{1}\right)$. This is true for $f_{1}$ by assumption. It is easily checked for the second term because the integration domain is compact and because the analyticity domain of $f_{1}$ depends only on 
the variables $p_{1}, p_{2}, p_{3}, p_{4}$. Finally, it is obvious for the last term which is, as in (6), the product of a pole $\frac{1}{k_{2}-\mu^{2}+i \varepsilon}, k=p_{1}+p_{2}-p_{4}$, by an analytic function in $\mathscr{R}_{1}$. Lemma 2 of Sect. 2.1 then entails that $h_{1}$ is analytic in $\mathscr{R}_{1}$.

(iii) Finally one checks easily from the structure theorem, applied to the bubble diagram function $\overline{(\mathrm{H}}_{1} \Theta_{-}{ }^{4}$, that this last term is then also necessarily analytic in $\mathscr{R}_{1}$ after factorization of its $\delta^{4}$-function [Remarks (i) and (ii) of Appendix 1 can be applied to see it].

Remark. The elimination, in step (ii), of the possible $\hat{u}_{+}$-singularities of $h_{1}$ along $\Omega_{+} U \mathscr{N}$ is crucial. Otherwise some of these singularities would propagate in this last step all along $L^{+}\left(G_{1}\right)$, and no result would be obtained anywhere.

We end this subsection, as announced, with a proof of Lemma 3. We give it in a form which can be directly adapted to the similar problems encountered in Sect. 3.2.

Let $D^{\prime}={ }_{3}^{\oplus} \Theta^{4}$ and let $D^{\prime}=D_{+}^{\prime}+\hat{D}^{\prime}$ be the decomposition of $D^{\prime}$ which is the analogue of the decomposition $D_{1}=D_{1}^{+}+\hat{D}_{1}$ (see Sect. 2). We have:

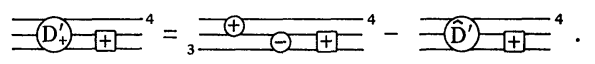

By two-particle unitarity, the first term in the r.h.s. of (14) is equal to $D_{1}$ and we therefore obtain:

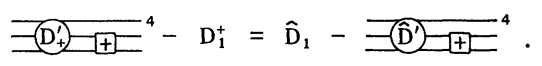

From the structure theorem (Appendix 1), one sees easily that the essential support of the terms in the 1.h.s. of (15) (after factorization of their $\delta^{4}$-functions), resp. in the r.h.s. of $(15)$, contains only $\hat{u}_{+}\left(P ; G_{1}\right)$ at any $P$ of $L^{+}\left(G_{1}\right)$ in $\mathscr{R}_{1}$ and is empty if $P \notin L^{+}\left(G_{1}\right)$, resp. does not contain $\hat{u}_{+}\left(P ; G_{1}\right)$ at any $P$ of $L^{+}\left(G_{1}\right)$ in $\mathscr{R}_{1}$. From the equality (15), the essential support of both sides is thus empty, i.e. analyticity is obtained, at all points $P$ in $\mathscr{R}_{1}$. This result, applied to the 1.h.s., is Lemma 3.

\subsection{Triangle Graphs}

We next consider a triangle graph, such as $\mathrm{G}_{2}=2$ Eq. (10) in the form:

$$
F_{2}-\exists \mathrm{F}_{\beta_{1}}=\Theta-{ }_{6}-\sum_{i=2,3} \exists \mathrm{F}_{\beta_{i}}=\Theta-{ }_{6}=H_{2},
$$

where $F_{\beta_{1}}, F_{\beta_{2}}, F_{\beta_{3}}$ are contributions to ${ }_{3}^{\frac{1}{2}} \overline{-}\left(+E_{8}^{6}\right.$ associated with the graphs
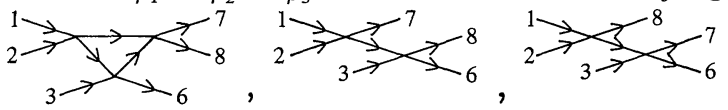

The internal lines of the second and third terms in the l.h.s. of (16) are here labelled 7,8: these terms are equal to

$$
\begin{gathered}
\frac{1}{2 !} \int F_{\beta_{i}}\left(p_{1}, p_{2}, p_{3} ; p_{7}, p_{8}, p_{6}\right) \times\left(S^{-1}\right)_{2,2}^{c}\left(p_{7}, p_{8} ; p_{4}, p_{5}\right) \\
\left.\cdot \prod_{\ell=7,8} \delta\left(p_{\ell}^{2}-\mu^{2}\right) \theta\left(p_{\ell}\right)_{0}\right) d^{4} p_{\ell}
\end{gathered}
$$


where the factor $1 / 2$ ! comes from the fact that there is a set of two lines between two common bubbles. Finally $\mathrm{H}_{2}$ is the sum of all terms in the l.h.s. of (10), except that $\exists(+)$ and $\overline{(+)_{-}}$are replaced by

$$
\sum_{\beta \neq 2} F_{\beta}-A \text { and } \sum_{\substack{\beta \\ \beta \neq \beta_{1}, \beta_{2}, \beta_{3}}} \overline{\mathrm{F} \beta} \boldsymbol{\Theta}_{6}+\overline{\mathrm{A}}_{6} .
$$

The terms singled out with $F_{2}$ in the 1.h.s. of (16) are again those whose singularities, at least along $L^{+}\left(G_{2}\right)^{8}$, correspond to diagrams with a related topological structure. After factorization of their $\delta^{4}$-functions, they have, like $f_{2}$, only $\hat{u}_{+}\left(P ; G_{2}\right)$ in their essential support at any $P$ of $L^{+}\left(G_{2}\right)$ in $\mathscr{R}_{2}$, and are analytic outside $L^{+}\left(G_{2}\right)$ in a subdomain $\mathscr{R}_{2}^{\prime}$ of $\mathscr{R}_{2}$ which contains $L^{+}\left(G_{2}\right) \cap \mathscr{R}_{2}$.

By "multiplication" on the right by $\beth^{ \pm}$and two-particle unitarity, one obtains in turn from (16):

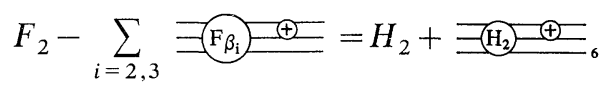

and hence:

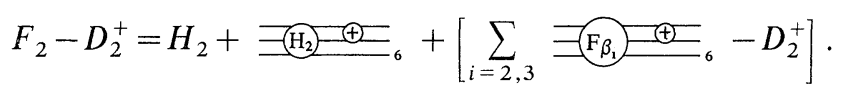

We show below that the three terms in the r.h.s. of (19) are analytic (after factorization of their $\delta^{4}$-functions) all along $L^{+}\left(G_{2}\right) \cap \mathscr{R}_{2}$. Since the terms $f_{2}$ and $d_{2}^{+}$of the 1.h.s. are known to be analytic outside $L^{+}\left(G_{2}\right)$ in $\mathscr{R}_{2}, f_{2}-d_{2}^{+}$will thus be analytic in $\mathscr{R}_{2}$. Q.E.D.

(i) The study of $\mathrm{H}_{2}$ is analogous to that of $H_{1}$ in Sect. 3.1. We do not give here the details. Several terms in $\mathrm{H}_{2}$ (after factorization of their $\delta^{4}$-functions) do contain $\hat{u}_{+}\left(P ; G_{2}\right)$ in their essential support at various points $P$ of $L^{+}\left(G_{2}\right)$ in $\mathscr{R}_{2}$ (see Remark below), but the union of these points does not cover $L^{+}\left(G_{2}\right) \cap \mathscr{R}_{2}$. By the same arguments as in Sect. 3.1, $h_{2}$ is therefore analytic at the other points of $L^{+}\left(G_{2}\right) \cap \mathscr{R}_{2}$.

(ii) The three terms in the 1.h.s. of (16) (after factorization of their $\delta^{4}$-functions), and hence $h_{2}$, satisfy the no sprout property at all points $P$ of $L^{+}\left(G_{2}\right)$ in $\mathscr{R}_{2}$. This is true for $f_{2}$ by assumption. It is shown for the second term in the same way as in Sect. 3.1. We prove it below for the last term (Lemma 4).

Lemma 2 of Sect. 2 therefore ensures that $h_{2}$ is analytic in $\mathscr{R}_{2}^{\prime}$.

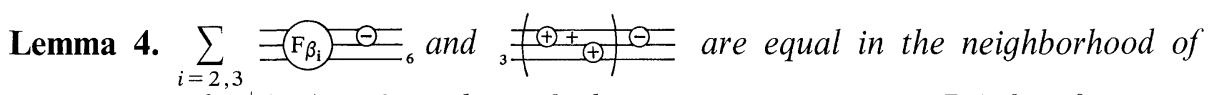
any point $P$ of $L^{+}\left(G_{2}\right)$ in $\mathscr{R}_{2}$ and satisfy the no sprout property at $P$ (after factorization of their $\delta^{4}$-functions).

8 The last term has singularities in $\mathscr{R}_{2}$ outside $L^{+}\left(G_{2}\right)$ corresponding to representations $\mathscr{D}$ of $G_{2}$ in which the time ordering conditions of diagrams $\mathscr{D}_{+}$are not satisfied for one at least of the internal lines incoming at the vertex where 4,5 are outgoing. An alternative proof, closer to that of Sect. 3.1, can be obtained by replacing this term by terms in the r.h.s. of (16), then denoted $H_{2}^{\prime}$. The r.h.s. of (19) is then simply $H_{2}^{\prime}+{ }_{-\left(H_{2}\right)} \oplus_{-}$. In contrast to $\mathrm{H}_{2}$ and $=$, these two terms can be shown to be analytic in the whole region $\mathscr{R}_{2}$ (after factorization of their $\delta^{4}$-functions). This is seen for $H_{2}^{\prime}$ by the same methods as below and by a straightforward adaptation of Lemma 5 
Proof. If $\mathscr{N}(P)$ is a sufficiently small neighborhood of $P$, one checks that the results of Sect. 3.1 apply to $F_{\beta_{i}}, i=2,3$, for all points $p_{7}, p_{8}$ in the integration domain. In fact one has $p_{1} \neq p_{2}$, and also $p_{8} \neq P_{6}$ or $p_{7} \neq p_{6}$, if $\left(P_{1}, P_{2}, P_{3} ; p_{7}, p_{8}, P_{6}\right)$ lies on $L^{+}\left(G_{\beta_{2}}\right), i=2,3$. This is because $P_{3} \neq P_{6}$ if $P \in L^{+}\left(G_{2}\right)$.

The two terms obtained for $i=2,3$ are equal, and their sum is thus equal,

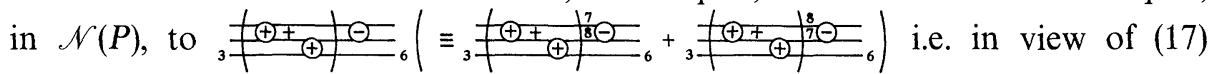
and (6) to:

$$
\begin{aligned}
I\left(p_{1}, \ldots, p_{6}\right)= & \delta^{4}\left(p_{1}+p_{2}+p_{3}-p_{4}-p_{5}-p_{6}\right) \\
& \cdot \int \frac{b\left(p_{1}, p_{2}, p_{3}, p_{7}, p_{8}, p_{6}\right)}{k^{2}-\mu^{2}+i \varepsilon} \times f_{2,2}^{(-)}\left(p_{7}, p_{8}, p_{4}, p_{5}\right) \\
& \cdot \delta^{4}\left(p_{7}+p_{8}-p_{4}-p_{5}\right) \prod_{\ell=7,8} \delta\left(p_{\ell}^{2}-\mu^{2}\right) \theta\left(\left(p_{\ell}\right)_{0}\right) d^{4} p_{\ell},
\end{aligned}
$$

where $k=p_{1}+p_{2}-p_{7}$, and $b$ and $f_{2,2}^{(-)}$are both analytic in the integration domain. [This is true for $f_{2,2}^{(-)}$because $(2 \mu)^{2}<\left(p_{4}+p_{5}\right)^{2}<(3 \mu)^{2}$.] In view of the analyticity of $b$ and $f_{2,2}^{(-)}$, the proof that the integral in the r.h.s. of (20) satisfies the no sprout property is a particular case of the study of Landau singularities of [21]. The integration domain is the sphere $S_{(2)}$ defined by $p_{7}^{2}=\mu^{2},\left(p_{4}+p_{5}-p_{7}\right)^{2}=\mu^{2}$, after elimination of $\delta$-functions. For real points $p$ on the non physical side of $L^{+}\left(G_{2}\right)$, $k^{2}-\mu^{2}$ does not vanish ${ }^{9}$, and hence the integrand is analytic. For complexified values of $p$, in the neighborhood of $P$, the integral will thus remain analytic away from the pinching locus of the cycle $\Gamma(p)$ (distorted from $S_{(2)}$ ) with the polar manifold $\pi(p)$ [defined by $\left(p_{1}+p_{2}-p_{7}\right)^{2}=\mu^{2}$ ]. This locus is just the complexified Landau variety. The no sprout property is thus in particular proved ${ }^{10}$.

(iii) As in Sect. 3.1, the above analyticity of $h_{2}$ ensures the analyticity of the term $=\left(\mathrm{H}_{2}\right)-{ }_{6}$ (after factorization of its $\delta^{4}$-function) in $\mathscr{R}_{2}^{\prime}$. The elimination in step (ii) of the possible $\hat{u}_{+}$-singularities of $h_{2}$ that remained in step (i) at some points is crucial: these $\hat{u}_{+}$-singularities would otherwise propagate all along $L^{+}\left(G_{2}\right)$ since points of $\Omega\left(G_{2}\right)$ are always encountered in integration domains.

(iv) We finally show:

Lemma 5. $\left[\sum_{i=2,3}=\mathrm{F}_{\beta_{i}} \oplus_{6}^{+}-D_{2}^{+}\right]$is analytic in the neighborhood of any point $P$ of $L^{+}\left(G_{2}\right)$ in $\mathscr{R}_{2}$ (after factorization of its $\delta^{4}$-function).

Proof. For the same reasons as in Lemma 4, the term $\sum_{i=2,3}=\mathrm{F}_{\beta_{i}}={ }^{\oplus}$ is equal to $\left.\left.{ }_{3}\right|^{\mid \oplus \pm_{+}}\right|^{(\oplus)}$ in the neighborhood of any $P$ of $L^{+}\left(G_{2}\right)$ in $\mathscr{R}_{2}$.

9 Being given the $+\alpha$-Landau surface of a graph $G$, here $G_{2}$, it divides locally the physical region into two parts, called its physical and non physical sides. It is well known [21] that, given a point $p$ on the non physical side, one cannot find on-mass-shell four-momenta for the internal lines of $G$ such that energy-momentum conservation be satisfied at each vertex of $G$. If $k^{2}$ could be equal to $\mu^{2}$, this would be possible

10 In the local coordinate system where $L^{+}\left(G_{2}\right)$ is defined by $q_{1}=0$ (see Sect. 2.1), the complexified Landau variety is defined by $\underline{q}_{1}=0$. Hence one has locally analyticity in particular whenever $\operatorname{Im} \underline{q}_{1}>0$ 


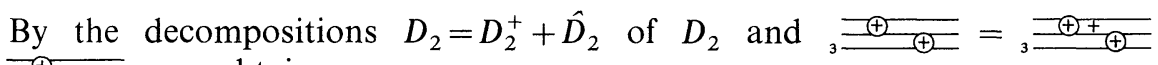

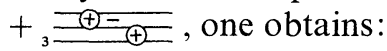

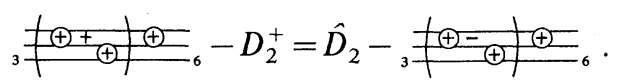

By the structure theorem (Appendix 1), the essential support at $P$ of the terms in the 1.h.s. (after factorization of their $\delta^{4}$-functions), resp. in the r.h.s., contains only $\hat{u}_{+}\left(P ; G_{2}\right)$, resp. does not contain it. Hence the essential support at $P$ of both sides is empty. Q.E.D.

This ends the proof of the theorem.

Remark. An example of points $P$ of $L^{+}\left(G_{2}\right)$ where several terms in $H_{2}$ do have $\hat{u}_{+}\left(P ; G_{2}\right)$ in their essential support (after factorization of their $\delta^{4}$-functions) is the set $\Omega\left(G_{2}\right)$ defined in Sect. 2.1. The corresponding terms in $=\left(\mathrm{H}_{2}\right)-\left(\oplus{ }_{6}{ }_{6}\right.$ have $\hat{u}_{+}\left(P ; G_{2}\right)$ in their essential support at all points $P$ of $L^{+}\left(G_{2}\right)$ because points of $\Omega\left(G_{2}\right)$ are always encountered in the integration domain.

The above terms in $H_{2}$ are the contributions $F_{\beta}$ to $=(+$ associated with the

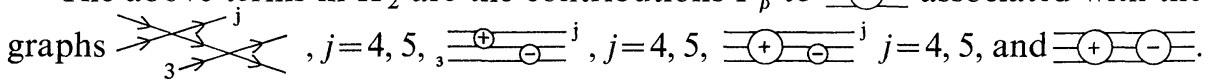
Examples of contributions to these terms that are at the origin of the $\hat{u}_{+}\left(P ; G_{2}\right)$ singularity at points of $\Omega\left(G_{2}\right)$ are the following classes, each of which corresponds to singularities with a related topological structure (similar classes are obtained by exchanging the roles of 4 and 5).

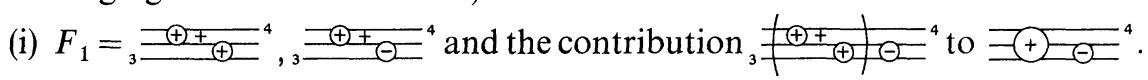
(We have made use here of the result of Sect. 3.1.)

(ii) The contributions to $\overline{\exists+\Theta^{-}}$arising from the contributions to $\exists(+$ corresponding to the graphs , and the two contributions to

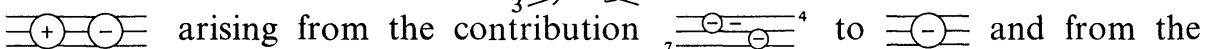
contributions to $=$ Ecorresponding to the graphs (We have denoted here by 7 one of the internal lines and have left the other ones implicit.)

The fact that the singularities of the terms of class (i) cancel among themselves can be checked directly. After the results of the present subsection have been obtained, this can also be checked for class (ii): this is a particular case of the results of Appendix B of [13].

\section{Appendix 1: The Structure Theorem}

Being given a bubble diagram function $F_{B}=f_{B} \times \delta^{4}\left(\sum_{i \in I(B)} p_{i}-\sum_{j \in J(B)} p_{j}\right)$, whose bubbles are not necessarily connected kernels of $S$ or $S^{-1}$ but may represent more general distributions $F_{b}=f_{b} \times \delta^{4}\left(\sum_{i \in I(b)} p_{i}-\sum_{j \in J(b)} p_{j}\right)$, the structure theorem of [11] (see also $[9,15]$ ) ensures that, away from $u=0$ points of $B, F_{B}$ (and $f_{B}$ ) are well defined and that the essential support of $F_{B}$, resp. of $f_{B}$, is contained in the set 
determined by the configurations, resp. the relative configurations (modulo global space-time translations), of external trajectories of diagrams $\mathscr{E}_{B}$. A diagram $\mathscr{E}_{B}$ is a collection of subdiagrams $\mathscr{E}_{b}$ that are associated with each bubble $b$ of $B$ and fit together. Each $\mathscr{E}_{b}$ is a configuration of trajectories associated with the incoming and outgoing lines of the bubble $b$, and corresponding to a point in the essential support of $F_{b}$. The subdiagrams $\mathscr{E}_{b}$ fit together if, being any internal line of $B$ that runs between two bubbles $b_{1}, b_{2}$, the corresponding trajectories in $\mathscr{E}_{b_{1}}$ and $\mathscr{E}_{b_{2}}$ coincide.

A point $P=\left\{P_{k}\right\}$ is a $u=0$ point of $B$ if there exists a $u=0$ diagram $\mathscr{E}_{B}(P)$ whose external four-momenta are $P_{k}$ and such that all external, but not all internal, trajectories pass through the origin.

When the bubbles $F_{b}$ are plus or minus bubbles representing respectively connected kernels of $S$ or of $S^{-1}=S^{\dagger}$, the diagram $\mathscr{E}_{b}$ are, according to macrocausality $[3,4]$, the configurations of external trajectories of diagrams $\mathscr{D}_{b}$. The latter are classical multiple scattering diagrams $\mathscr{D}_{+}$(with possibly vertices at infinity: see $[8,9]$ ) if $b$ is a plus bubble, or (by unitarity) opposite diagrams $\mathscr{D}_{-}$ if $b$ is a minus bubble. This is still true if a bubble $b$ represents a contribution $\left(F_{\beta}\right)_{b}$ to a connected kernel of $S$, or $S^{-1}$ associated with a given $+\alpha$-Landau surface $L^{+}\left(G_{\beta}\right)$. The only $\mathscr{D}_{b}$ to be considered are then those whose topological structure is $G_{\beta}$. The diagrams $\mathscr{E}_{B}$ can thus be replaced by diagrams $\mathscr{D}_{B}$, which are collections of subdiagrams $\mathscr{D}_{b}$ that fit together in the same sense as above.

We end with the following remarks:

(i) Saying that $f_{b}$, or $f_{B}$, is analytic at a point $P$ is equivalent to saying that all points in $E S_{P}\left(F_{b}\right)$, or $E S_{P}\left(F_{B}\right)$, correspond to configurations of external trajectories that all pass through a common point.

(ii) In the applications to Sects. 2 and 3, the incoming center-of-mass energy $s_{b}^{1 / 2}$ to each bubble $=+=$ or $=-$ = is strictly less than $3 \mu$ and (most of the time) strictly larger than $2 \mu$. Its essential support then corresponds, by macrocausality (and unitarity) to external trajectories that all pass through a common point, i.e. external trajectories of trivial diagrams $\mathscr{D}_{b}$ with only one vertex. (This vertex may be at infinity of $s_{b}^{1 / 2}=2 \mu$, i.e. if all four-momenta are parallel.)

\section{Appendix 2: Previous Derivations of the Pole-Factorization Theorem}

We restrict our attention, as in Sect. 3 , to a $3 \rightarrow 3$ process below the four-particle threshold, and consider here a point $P$ of the surface $L^{+}\left(G_{1}\right)$ that lies on no other $+\alpha$-Landau surface. The pole-factorization theorem then asserts that $f=d_{1}^{+}$, modulo an analytic function, in the neighborhood of $P$.

\section{Proof of [1]}

The proof of [1] starts from (10) written in the form:

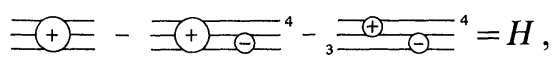

where $H$ is the sum of all terms in the l.h.s. of (10) apart from the three terms singled out in the 1.h.s. of (22).

It is asserted in [1] (without proof) that all terms in $H$, after factorization of their $\delta^{4}$-functions, are either analytic or (like $\exists-$ E) minus $i \varepsilon$ boundary values 
of analytic functions along $L^{+}\left(G_{1}\right)$. If we put $H=h \times \delta^{4}\left(p_{1}+p_{2}+p_{3}-p_{4}-p_{5}-p_{6}\right)$, then $h$ is itself along $L^{+}\left(G_{1}\right)$ a minus $i \varepsilon$ boundary value. If this was proved, the pole-factorization theorem would follow easily. The method we present here is different from the original proof of [1], but the discussion presented below applies equally in both cases.

Equation (22) is transformed by "multiplication" on the right of all terms by $\overline{\Xi \pm \mp}^{4}$ and two-particle unitarity into:

or

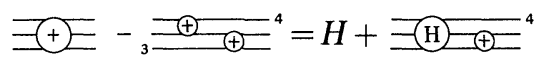

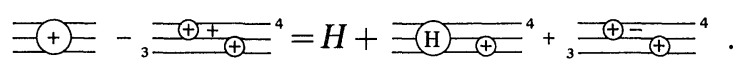

The previous "result" on $h$ yields an analogous result for the term $=(\mathrm{H})-\left(+{ }^{4}\right.$ and an easy argument (for instance the edge-of-the-wedge theorem) allows one to conclude.

However, the above assertion on the terms that occur in $H$ is not correct. Several terms are not minus $i \varepsilon$ boundary values, and moreover do have $\hat{u}_{+}\left(P ; G_{1}\right)$ in their essential support at various points $P$ of $L^{+}\left(G_{1}\right)$ : see [15]. Some of these $\hat{u}_{+}$-singularities propagate all along $L^{+}\left(G_{1}\right)$ in the final step.

Remark. In contrast to $h_{1}$ in Sect. $3.1, h$ is itself expected to contain $\hat{u}_{+}\left(P ; G_{1}\right)$ in its essential support at the points $P$ of the open subset $\Omega_{+}$of $L^{+}\left(G_{1}\right)$. In fact $H$ and $H_{1}$ differ in particular by $\sum_{i=1,2}=\mathrm{F}_{\beta_{i}}={ }^{4}$ where the $F_{\beta_{i}}$ correspond here to triange graphs support along $\Omega_{+}$.

\section{Proof Using the Assumption of Separation of Singularities}

We use below (23) or (24). The argument of $[10,12]$ makes use of a somewhat different equation, but is completely similar. The result is directly obtained from the assumption of separation of singularities because the terms $\bar{\exists}\left(\simeq F_{1}\right.$ near $\left.P\right)$ and ${ }_{3}=\frac{\left(++_{+}\right.}{\oplus}$ are the only ones whose singularities correspond to diagrams with a structure related to that of the diagram $\mathscr{D}_{+}\left(P ; G_{1}\right)$. For present purposes, the assumption can also be stated, as in $[10,12]$ as an assumption of "mixed- $\alpha$ cancellation". The proof is completely disrupted if this assumption is dropped,

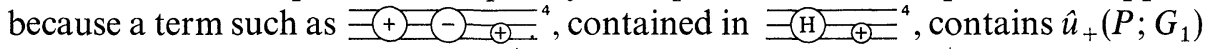
in its essential support all along $L^{+}\left(G_{1}\right)$ : being given any $P$ in $L^{+}\left(G_{1}\right)$, there exists a $\mathscr{D}_{B}(P)$ whose configuration of external trajectories coincides with that of $\mathscr{D}_{+}\left(P ; G_{1}\right)$, although its structure is different (see [15]).

\section{Proof of [15]}

The proof of [15] is analogous to that of Sect. 3.1, but makes use of (22) and (23) [or (24)], rather than (11) and (12). Although $f=f_{1}$ modulo an analytic function near the point $P$ of interest, the replacement of ${\overline{\nexists\left(F_{1}\right)}-E^{4}}^{4}$ by ${\overline{\exists+E^{-}}}^{4}$ in (22) is the source of several complications arising from the occurrence of points that belong to other $+\alpha$-Landau surfaces in the integration domain. In this connection, see in particular the remark that concludes the above Subsect. 1 of this appendix. 


\section{Appendix 3: Possible Extensions of the Results}

\section{No Sprout Assumption}

The no sprout assumption of Sect. 2.1 extends with no change if related $+\alpha$-Landau surfaces are not encountered. Otherwise, it has to be modified in the neighborhood of points $P$ where related $+\alpha$-Landau surfaces are involved.

A general form of the no sprout assumption may be the regularity property $R$ of [9]. This problem requires, however, further study.

\section{Generalized Feynman Integrals}

The definition of the generalized Feynman integrals given in Sect. 2.2, extends in a similar way to more general simple graphs $G$ (i.e. graphs with no set of more than one internal line between any pair of vertices), in regions in which $L^{+}(G)$ is not related to other $+\alpha$-Landau surfaces. Modifications are needed otherwise. For a particularly simple example (involving only simple graphs), see Appendix C of [13], or [16].

\section{Extensions of the Results of Section 3}

We shall only outline below a tentative extension of Sect. 3.1 to the region $\left(4 \mu^{2}\right)<s$ $<(5 \mu)^{2}, p \notin \mathscr{M}_{0}$. The analogue of $(11)$ is:

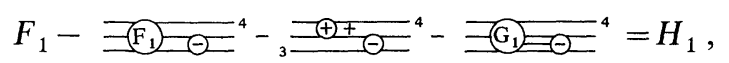

where $G_{1}$ is the contribution to $\exists+E$ associated with the graph and $H_{1}$ contains several new terms [which vanish when $s<(4 \mu)^{2}$ ].

In view of the presence of the last term in the 1.h.s. of (25), we also consider the analogue of $(25)$ for a $3 \rightarrow 4$ process:

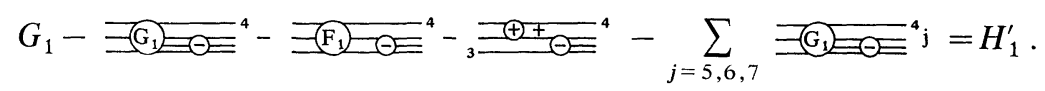

By "multiplication" on the right of all terms of (25) by ${\overline{\bar{A}^{+}}}^{4}$, of (26) by $\overline{\equiv \oplus}^{4}$ $\left(\equiv \overline{\equiv \oplus \Sigma}^{4}\right.$ ), substraction of the equations obtained, and by unitarity for $3 \rightarrow 2$ processes, one obtains:

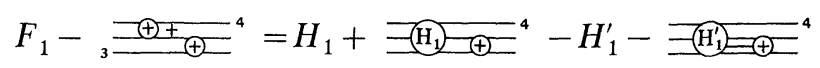

modulo an analytic function (after factorization of the $\delta^{4}$-functions).

The desired result follows if one can show that the four terms in the r.h.s. of (27) are analytic (after factorization of $\delta^{4}$-functions). An actual proof will require more detailed studies, including possibly a more systematic use of the regularity property $R$ of [9]. In fact, problems arise for instance for the proof of the no sprout property for the term $\equiv \mathrm{G}_{1}=\sigma^{4}$, because $\mathscr{M}_{0}$ points are always encountered in the integration domain. Similar, possibly more delicate, problems arise in the study of $\exists\left(G_{1}\right)=\Theta^{4}$ and $\exists\left(G_{1}=\Theta^{4}\right.$. Finally, the proof of analyticity for the term $\left.\exists \mathrm{H}_{1}^{\prime}\right)=\mathrm{E}^{4}$ requires, again for the same reason, the previous determination of the essential support of $h_{1}^{\prime}$ at $\mathscr{M}_{0}$ points. 


\section{References}

1. Eden, R.J., Landshoff, P.V., Olive, D.I., Polkinghorne, J.C.: The analytic $S$ matrix, Chap. IV and references therin. Cambridge: Chambridge University Press 1966

Bloxham, M., Olive, D.I., Polkinghorne, J.C.: J. Math. Phys. (N.Y.) 10, 494, 545, 553 (1969) Olive, D.I.: In: Hyperfunctions and theoretical physics, Lectures Notes in Mathematics, p. 133. Berlin, Heidelberg, New York: Springer 1975

2. Chew, G.F.: The analytic $S$ matrix. New York: W. A. Benjamin, 1966

3. Iagolnitzer, D., Stapp, H.P.: Commun. Math. Phys. 14, 15 (1969)

4. Iagolnitzer, D.: The $S$ matrix, Chap. II and references therein. North-Holland. 1978

5. Iagolnitzer, D.: In: Structural analysis of collision amplitudes (eds. R. Balian, D. Iagolnitzer) Part III, p. 295 and references therein. Amsterdam: North-Holland 1976

6. For a complete proof, see Bony, J.M., in Goulaouic-Schwartz Séminaire, Paris (1976)

7. Sato, M., Kawai, T., Kashiwara, M.: Microfunctions and pseudo-differential equations, Lecture Notes in Mathematics, p. 265. Berlin, Heidelberg, New York: Springer 1973

8. Kashiwara, M., Kawai, T., Stapp, H.P.: Commun. Math. Phys. 66, 95 (1979)

9. Iagolnitzer, D.: Commun. Math. Phys. 63, 49 (1978)

10. Coster, J., Stapp, H.P.: J. Math. Phys. (N.Y.) 10, 371 (1969); 11, 1441 (1970); 11, 2753 (1970)

11. Iagolnitzer, D.: Commun. Math. Phys. 41, 39 (1975)

12. Stapp, H.P.: In: Structural analysis of collision amplitudes (eds. R. Balian, D. Iagolnitzer), p. 275. Amsterdam: North-Holland 1976

13. Iagolnitzer, D.: The $S$ matrix, Chap. III. Amsterdam: North-Holland 1978

14. Kashiwara, M., Kawai, T.: In: Complex analysis, microlocal calculus, and relativistic quantum theory, Lecture Notes in Physics 126, p. 5 and references therein. Berlin, Heidelberg, New York: Springer 1980

15. Iagolnitzer, D., Stapp, H.P.: Commun. Math. Phys. 57, 1 (1977)

16. Iagolnitzer, D.: In: Complex analysis, microlocal calculus, and relativistic quantum theory, op. cit. in [14], and references therein

17. Bros, J.: Cours de Lausanne, May 1979 (in preparation)

18. Epstein, H., Glaser, V., Iagolnitzer, D.: In preparation

19. Bros, J., Epstein, H., Glaser, V.: Helv. Phys. Acta 45, 149 (1972)

For a more direct version, see also Appendix III of Part IV of Iagolnitzer, D.: The $S$ matrix, op. cit. in [4]

20. Kawaï, T., Stapp, H.P.: Publ. R.I.M.S. Kyoto Univ. 12 (Suppl.), 155 (1977)

21. Pham, F.: Introduction à l'étude topologique des singularités de Landau. In: Memorial des Sciences Mathématiques, Fasc. CLXIV. Paris: Gauthier-Villars 1967. For the study of analogous integrals in general field theory, see [17] and Bros, J., Katz, A. (in preparation)

Communicated by R. Stora

Received July 5, 1979; in revised form February 11, 1980 
